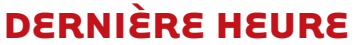

\section{Cancer pulmonaire}

\section{Rôle des TLR7 et TLR8 dans la survie cellulaire et la résistance à la chimiothérapie}

Julien Cherfils-Vicini, Diane Damotte, Wolf-Herman Fridman, Catherine Sautès-Fridman, Isabelle Cremer

\section{Lien entre l'inflammation}

\section{et les cancers pulmonaires}

De nombreuses données épidémiologiques et expérimentales démontrent qu'il existe un lien entre l'inflammation chronique, la carcinogenèse et la progression tumorale [1]. Dans les poumons, l'inflammation chronique peut être provoquée par des infections virales ou bactériennes répétées, par une exposition prolongée à des agents toxiques tels que le tabac ou l'amiante, ou être liée à des maladies respiratoires chroniques. Cette inflammation chronique pulmonaire peut augmenter le risque de carcinogenèse $[2,3]$. Une des molécules clés de l'inflammation est le facteur de transcription $N F-\kappa B$, dont la fonction dans le développement tumoral dans un contexte d'inflammation chronique a été démontrée $[4,5]$. NF- $K B$ est un des partenaires des voies de signalisation en aval des récepteurs Toll-like (TLR), récepteurs membranaires ou intracellulaires reconnaissant les molécules associées aux agents pathogènes (PAMP) et aux dommages cellulaires (DAMP) ${ }^{1}$ lors de la réponse immunitaire innée. Bien que l'expression des TLR ait initialement été observée dans les cellules du système immunitaire, plusieurs études l'ont décrite dans des cellules épithéliales tumorales. Ainsi l'expression de TLR4 [6] et de TLR9 [7] par les cellules tumorales pulmonaires humaines a été

${ }^{1}$ PAMP : Pathogen-associated molecular patterns; DAMP Damage-associated molecular patterns. décrite. Enfin, des études récentes ont impliqué certains TLR dans le développement tumoral au vu de leur capacité à réguler la croissance et la survie des cellules tumorales [8]. L'épithélium pulmonaire est la cible d'infections répétées par des virus comme le virus respiratoire syncytial ou le virus influenza. Ces virus appartiennent à la famille des virus à ARN simple brin qui sont reconnus par les TLR7 et 8 . Ainsi, les infections pulmonaires pourraient contribuer indirectement au développement des cancers pulmonaires.

\section{Expression des TLR7 et TLR8}

par les cellules tumorales pulmonaires Nous avons émis I'hypothèse que TLR7 et/ou TLR8 contribuent à l'inflammation dans les poumons, et que la signalisation par ces récepteurs peut favoriser la progression tumorale. Nous avons étudié par immunohistochimie l'expression de TLR7 et TLR8 sur des coupes de tumeurs provenant de patients opérés de carcinomes pulmonaires non à petites cellules (NSCLC, non-small cell lung carcinoma) [9]. Sur une petite cohorte de 13 patients, nous avons montré que TLR7 est exprimé chez 9 patients, et TLR8 chez les 13 patients, avec des pourcentages d'expression variables allant de moins de $10 \%$ à $100 \%$ de cellules tumorales exprimant TLR7 et/ou TLR8. Nous avons observé une forte expression de TLR7 (Figure IA) et de TLR8 (Figure IB) par les cellules tumorales d'adénocarcinomes et de carcinomes épidermoïdes. L'expression
J. Cherfils-Vicini, C. Sautès-Fridman, I. Cremer : Institut national de la santé et de la recherche médicale (INSERM), U872, Centre de recherche des Cordeliers,

Paris, F-75006, France ;

Université Pierre et Marie Curie-Paris 6, UMRS 872, Paris F-75006, France ;

Université Paris Descartes, UMRS 872 ,

Paris F-75006, France.

D. Damotte : Institut national de la santé et de la recherche médicale (INSERM), U872, Centre de recherche des Cordeliers,

Paris, F-75006, France ;

Université Pierre et Marie Curie-Paris 6, UMRS 872, Paris F-75006, France ;

Université Paris Descartes, UMRS 872,

Paris F-75006, France;

Service d'anatomo-pathologie,

Hôpital Hôtel Dieu, AP-HP,

Paris F-75005, France.

isabelle.cremer@crc.jussieu.fr

julien.cherfils@crc.jussieu.fr

W.H. Fridman : Institut national de la santé et de la recherche médicale (INSERM), U872, Centre de recherche des Cordeliers,

Paris, F-75006, France ;

Université Pierre et Marie Curie-Paris 6, UMRS 872, Paris F-75006, France;

Université Paris Descartes, UMRS 872 , Paris F-75006, France;

Service d'immunologie biologique, Hôpital Européen Georges Pompidou, AP-HP, Paris F-75015, France.

de TLR7 est périnucléaire alors que celle de TLR8 est cytoplasmique. L'expression de ces deux récepteurs a également été observée sur les cellules épithéliales bronchiques alors qu'elle ne l'est pas sur les cellules alvéolaires non tumorales.

La stimulation de TLR7 et TLR8

induit une augmentation

de la survie cellulaire

Pour démontrer la fonction des TLR dans les cellules tumorales, nous avons étudié in vitro l'activation des voies de signalisation en aval de TLR7 et 8 dans des cellules humaines A549 (adénocarcinome) et SKMES (carcinome épidermoïde). Nous 

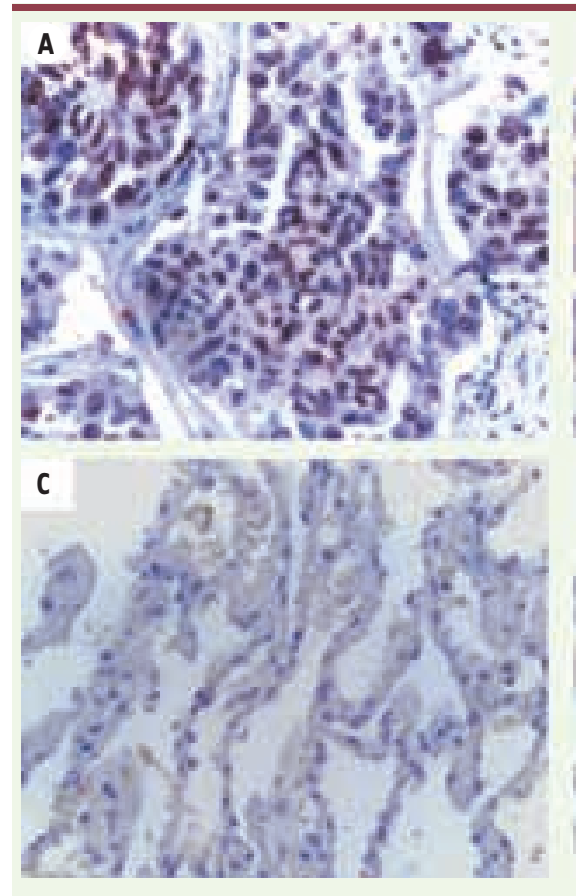

avons démontré que la stimulation des cellules par la loxoribine (analogue de la guanosine), ligand de TLR7 ou par le poly $U$, ligand de TLR8, induit une activation atypique de $N F-\kappa B$, accompagnée de la translocation des sous-unités p50 et p65 dans le noyau des cellules. Cette activation est dite atypique car $1 \kappa B \alpha$ est phosphorylé $30 \mathrm{~min}$ après la stimulation sans être dégradé. La stimulation des cellules tumorales pulmonaires par les ligands de TLR7 ou de TLR8 induit une augmentation de la survie des cellules, en accord avec une augmentation de l'expression de la protéine anti-apoptotique $\mathrm{Bcl}-2[9,10]$.

\section{La stimulation de TLR7 et TLR8 induit} une résistance à la chimiothérapie Les patients atteints de cancer pulmonaire sont souvent traités par polychimiothérapie associant des sels de platine avec une drogue de deuxième génération comme la navelbine [11]. Nous avons émis l'hypothèse que la stimulation des TLR7 et TLR8 exprimés par les cellules tumorales pouvait induire un état de résistance à la mort cellulaire induite par les drogues utilisées en chimiothérapie. Nous avons réalisé des expériences de formation de colonies de cellules tumorales stimulées

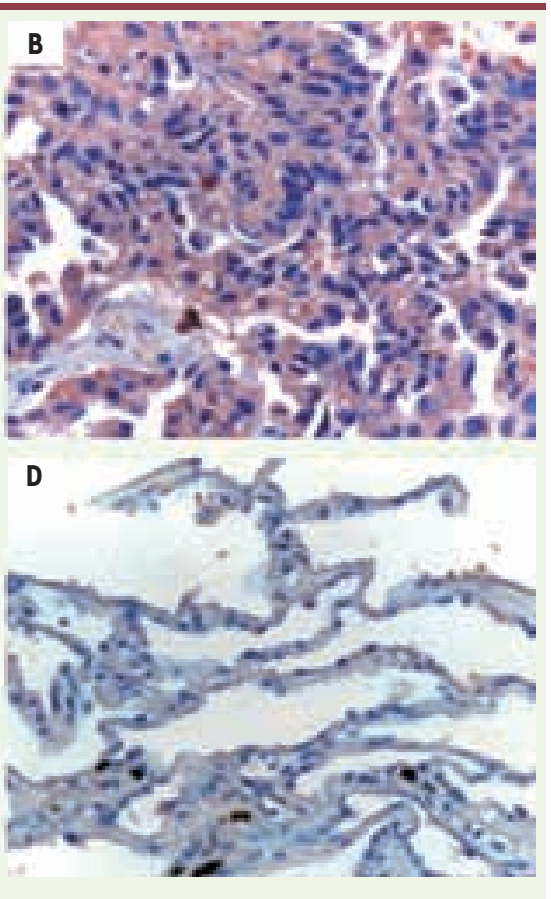

ou non par les ligands de TLR7 ou TLR8, et traitées par chimiothérapie (Figure 2). Nous avons ainsi montré que la stimulation des cellules par la loxoribine ou le polyU induit leur résistance à la chimiothérapie. Ainsi, lorsque les cellules sont stimulées par la loxoribine avant le traitement par chimiothérapie, la fraction de cellules survivantes est deux à trois fois supérieure à ce qu'elle est dans les expériences où les cellules exposées à la chimiothérapie ne sont pas stimulées par les ligands de TLR (Figures 2A, B). Cet état de résistance a été observé en réponse à une monochimiothérapie (Figure 2A) ou une polychimiothérapie (Figure 2B). On observe que la stimulation par des agonistes de TLR7 provoque une perte de sensibilité d'un facteur 100 à 1000 des cellules tumorales au cisplatine $[9,10]$.

\section{Profil d'expression génique} des cellules tumorales pulmonaires en réponse aux agonistes des TLR Dans les cellules du système immunitaire, la stimulation des TLR induit la production de cytokines inflammatoires. Nous avons analysé la modulation de l'expression de gènes codant des molécules effectrices de l'immunité et de l'angio-
Figure 1. Analyse par immunohistochimie de l'expression de TLR7 ( $A, C)$ et $\operatorname{TLR} 8(B, D)$. Coupes de tumeurs pulmonaires inclues en paraffine, analyse de cellules tumorales ( $A, B)$ ou de cellules épithéliales alvéolaires non tumorales (C-D). Agrandissement : $x 40$.

genèse dans les lignées cellulaires tumorales pulmonaires stimulées ou non par les ligands des TLR7 ou TLR8, ainsi que dans les cellules tumorales primaires isolées à partir de prélèvements de patients. La stimulation des cellules par les ligands de TLR7 ou TLR8 induit une augmentation de l'expression de certains gènes impliqués dans la survie cellulaire, l'angiogenèse et l'échappement au système immunitaire. Parmi ces gènes on retrouve une forte augmentation de $\mathrm{Bcl}-2$ et du récepteur de chimiokines CCR4.

Enfin, nous avons montré sur un nombre limité d'échantillons de tissus de patients que le profil d'expression de ces gènes par les cellules tumorales est identique à celui des lignées cellulaires qui ont été stimulées in vitro par la loxoribine ou le polyU. Ces résultats suggèrent fortement que les cellules tumorales des patients ont été stimulées in situ, dans la tumeur, par des ligands endogènes des TLR7 et TLR8, qui pourraient être des virus à $A R N$ ou des $A R N$ issus des cellules nécrotiques.

\section{Perspectives}

Un des problèmes en thérapeutique anticancéreuse est d'adapter le traitement aux caractéristiques de la tumeur du patient de façon à obtenir l'efficacité maximale avec un minimum de toxicité. La question est donc celle des critères de choix thérapeutique et de la reconnaissance des bons et mauvais répondeurs, notamment grâce à l'identification de biomarqueurs prédictifs de résistance à la chimiothérapie.

Plusieurs de ces biomarqueurs candidats sont actuellement en cours d'étude dans les cancers du poumon, dont les protéines MDR (multi-drug resistance). Il a par exemple été montré que MDR7/ABCC10 est un biomarqueur prédictif de résistance au paclitaxel (taxol) et à la vironelbine dans les cancers du poumon de type NSCLC, et qu'une forte 


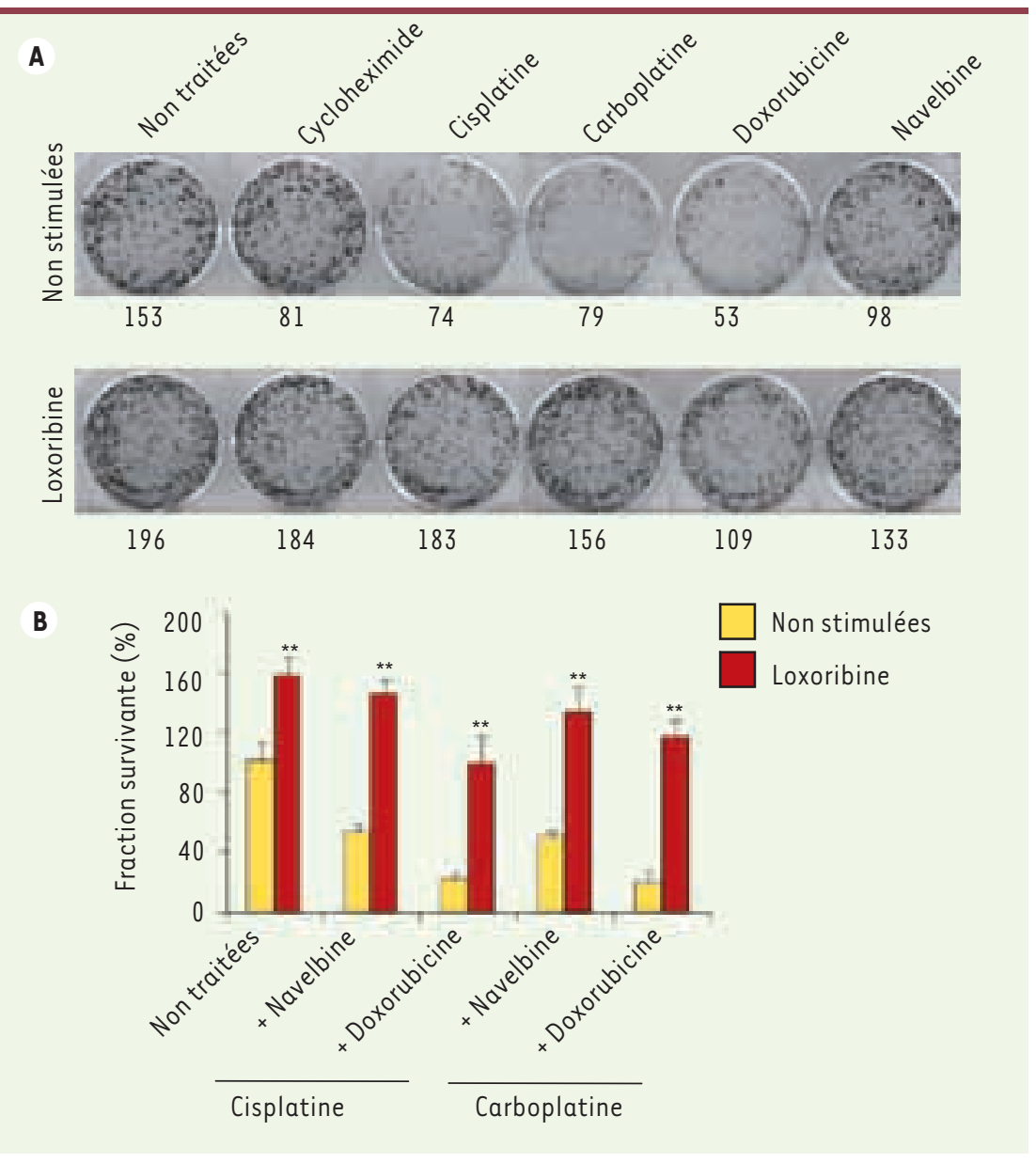

expression d'ABCC5² est corrélée à une résistance à la gemcitabine. Ces transporteurs reflètent la capacité intrinsèque des cellules tumorales à éliminer les drogues de chimiothérapie.

Cependant les tumeurs se développent dans un environnement inflammatoire dont les effets sur la réponse à la chimiothérapie restent inexplorés à ce jour et qui pourrait être un acteur important de la réponse à la chimiothérapie. Les TLR sont exprimés par les cellules du système immunitaire, et les données de la littérature indiquent que TLR7 et/ou TLR8 sont exprimés par les cellules dendritiques plasmacytoïdes ( $\mathrm{pDC}$ ) et myéloïdes $(\mathrm{mDC})$, les lymphocytes $B$ et les lymphocytes Natural Killer (NK). Nous avons également montré que les cellules des

${ }^{2}$ Protéines $A B C$ : famille des transporteurs membranaires à ATP binding cassette, qui utilisent l'hydrolyse d'ATP pour l'efflux de substrats. structures lymphoïdes tertiaires présentes dans les cancers pulmonaires de type NSCLC [12] expriment fortement TLR7 et plus faiblement TLR8. Une des approches d'immunothérapie anticancéreuse pourrait donc utiliser la stimulation des TLR. Toutefois, nos travaux incitent à la prudence : l'administration de ces agonistes doit prendre en compte l'expression de TLR7 et 8 par les cellules tumorales des patients, surtout si un traitement combinant agonistes des TLR et chimiothérapie est envisagé, afin d'éviter que ne se développe in situ un état de résistance à la chimiothérapie ou une augmentation de la survie des cellules tumorales. $\diamond$ Human lung cancer: role of TLR7 and TLR8 in cell survival and chemoresistance

\section{CONFLIT D'INTÉRÊTS}

Les auteurs déclarent n'avoir aucun conflit d'intérêts concernant les données publiées dans cet article.
Figure 2. La stimulation des cellules $A 549$ pendant $48 \mathrm{~h}$ avec la loxoribine induit une résistance à la chimiothérapie. Les cellules ont été stimulées aux jours 0, 3, 6 et 9 par la loxoribine, puis ont été traitées le jour 12 par les drogues de chimiothérapie, pendant 36h. La survie cellulaire a été mesurée par un test de formation de colonies ( $A$ ). La fraction survivante ( $B$ ) a été calculée ainsi: nombre de colonies après le traitement par chimiothérapie/nombre de cellules mises en culture au jour 0 × PE $\times 100$. PE est l'efficacité de formation de colonies calculée ainsi : nombre de colonies/nombre de cellules mises en culture au jour 0 .

\section{REMERCIEMENTS}

Ce travail a été financé par l'Inserm, l'Institut National du Cancer et l'Association pour la Recherche contre le Cancer (INCA/ARC R07120DP), l'Université Pierre et Marie Curie et l'Université Paris Descartes. Julien Cherfils a été financé par le cancéropôle lle de France et l'ARC.

\section{RÉFÉRENCES}

1. Coussens LM, Werb Z. Inflammation and cancer. Nature $2002 ; 420: 860-7$.

2. Littman AJ, Thornquist MD, White $\varepsilon$, et al. Prior lung disease and risk of lung cancer in a large prospective study. Cancer Causes Control 2004 ; 15 : 819-27.

3. Ardies CM. Inflammation as cause for scar cancers of the lung. Integr cancer ther $2003 ; 2$ : 238-46.

4. Karin M, Greten FR. NF-kappaB: linking inflammation and immunity to cancer development and progression. Nat Rev Immunol 2005 ; 5 : 749-59.

5. Pikarsky $\varepsilon$, Porat R, Stein I, et al. NF- $\kappa B$ functions as a tumour promoter in inflammation-associated cancer. Nature $2004 ; 431: 461-466$.

6. He W, Liu $Q$, Wang L, et al. TLR4 signaling promotes immune escape of human lung cancer cells by inducing immunosuppressive cytokines and apoptosis resistance. Mol Immunol 2007 ; 44 : 2850-9.

7. Droemann D, Albrecht D, Gerdes J, et al. Human lung cancer cells express functionally active Toll-like receptor 9. Respir Res $2005 ; 6: 1$.

8. Tsan MF. Toll-like receptors, inflammation and cancer. Semin Cancer Biol $2006 ; 16: 32-7$.

9. Cherfils-Vicini J, Platonova S, Gillard M, et al. Triggering of TLR7 and TLR8 expressed by human lung cancer cells induces cell survival and chemoresistance. J Clin Invest 2010 ; 8 mars : pli 36551. doi : 10.1172/JCl 36551.

10. Brevet Européen (demande déposée en août 2008): EP08305490. Methods for predicting the response to treatment and for treating cancer. Fridman WH, CherfilsVicini J, Cremer I. Extension PCT: PCT/EP2009/060555.

11. Spira A, Ettinger DS. Multidisciplinary management of lung cancer. N Engl J Med 2004 ; 350 : 379-92.

12. Dieu-Nosjean MC, Antoine M, Danel C, et al. Long-term survival for patients with non-small-cell lung cancer with intratumoral lymphoid structures. J Clin Oncol 2008 ; $26: 4410-7$.

\section{TIRÉS À PART}

I. Cremer 


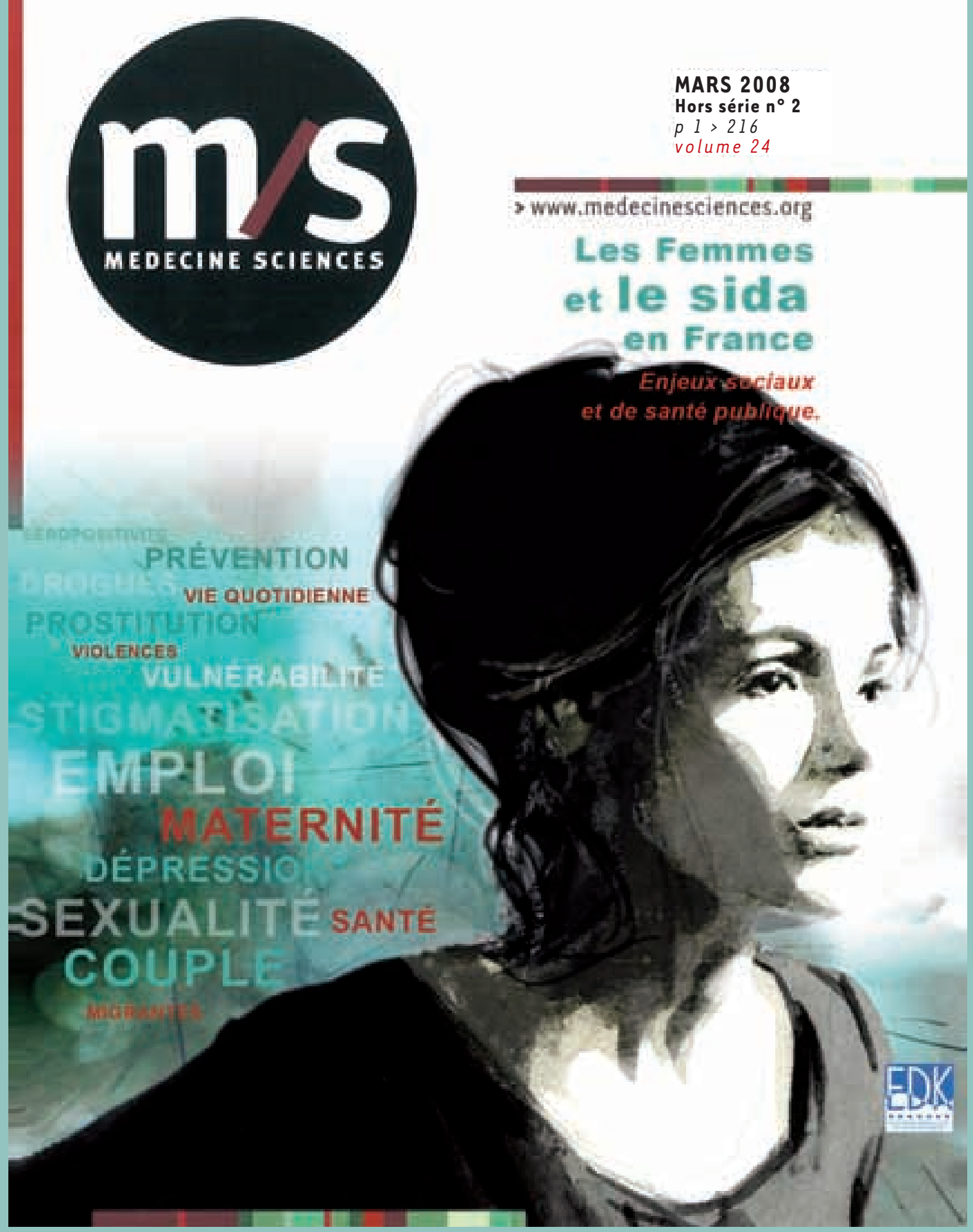

L'Anrs (Agence nationale de recherches sur le sida et les hépatites virales) publie « Les femmes et le sida en France - Enjeux sociaux et de santé publique ». Un numéro indispensable pour comprendre les nouveaux enjeux de prévention dans le contexte de féminisation et de précarisation de l'épidémie et au moment où se pose la question des conditions de vie des femmes atteintes par l'infection du VIH en France.

Un ouvrage précieux, destiné aux chercheurs, élus, décideurs en santé et représentants associatifs.

\section{Bon de commande}

À retourner à EDK, 2, rue Troyon - 92316 Sèvres Cedex

Tél. : 0155641393 - Fax : 0155641394 - E-mail : edk@edk.fr

NOM :

Prénom :

Adresse :

Code postal :

Ville :

Pays :

Fonction :

Je souhaite recevoir le hors série $n^{\circ} 2$ de $M / S$ Les femmes et le Sida en France: $18 €+3 €$ de port $=21 €$ TTC en ................. exemplaire, soit un total de $€$

$\square$ Par chèque, à l'ordre de $\mathbf{E} \mathbf{D} \mathbf{K}$

$\square$ Par carte bancaire : $\square$ Visa $\square$ Eurocard/Mastercard

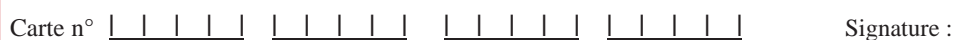

Date d'expiration: $\quad$ । 1 | 1 | |

$\mathrm{N}^{\circ}$ de contrôle au dos de la carte : $\quad$ ～～～| | 